\title{
Traffic Safety Evaluation of Urban Road Intersection Based on Grey Clustering
}

\author{
YUJingfei ${ }^{1, a^{*}}$, Wang $\mathrm{Li}^{2, \mathrm{~b}}$ \\ ${ }^{1}$ The School of Architecture and Civil Engineering, Inner Mongolia University of Science and \\ Technology, Baotou, 014010, China \\ 2 The School of Information Science and Technology, Baotou Teachers' College, Baotou, 014030 \\ ayjf_05025@126.com, bbtsfxywl@126.com
}

Keywords: Intersection; Traffic safety; Traffic conflict; Grey clustering; Evaluation

Abstract. For problems on impacting urban road traffic in view of traffic safety conditions of the urban road intersections, the paper evaluated the intersection safety levels by the traffic conflict technique and grey cluster analysis method. The result of the case analysis shows that the evaluation results are close to the actual operating conditions of the intersections. At the same time, it indicates that the method can be more objectively evaluate the safety conditions in the intersections.

\section{Introduction}

Urban road intersections are an important node to implement direction conversion of all kinds of vehicle and pedestrians. Due to the large accumulation of vehicles and pedestrians in intersection, therefore they easily lead to traffic accident and cause traffic congestion. According to relevant survey, the number of traffic accident around the world each year at the intersection accounted for about $10 \% \square 40 \%$ of the total traffic accident; the number of traffic accidents happened at the intersection in our country is approximately $30 \%$, while road capacity in intersection will reduce 40\% 50\% [1,2]. Therefore, to accurately grasp traffic safety condition of intersection has an important significance to improve intersection capacity and management level. Indicators often used mainly the number of accidents, the number of deaths and the direct economic loses etc. when people currently evaluate traffic safety level [3]. Due to the randomness of the traffic accidents, these indicators are often difficult to obtain. So the paper uses the traffic conflict data which are relatively easy to obtain as evaluation index, employ grey clustering method to evaluate traffic safety of intersection.

\section{Traffic conflict}

Traffic Conflict (TC) is a new method for rapid assessment on site traffic safety conditions; it is different from the traditional method that use accident index as the safety evaluation indexes [4]. The first academic annual meeting of the international traffic conflict is held in Oslo in 1997, standard definition of traffic conflict was officially proposed at the meeting. Two or more road users are close to a certain extent each other at a certain time and space, if they do not change the operating state, the risk of collision may occur, this phenomenon is called traffic conflict [5]. The essence of traffic conflict is the expression form of in-security transportation behavior and is a potential accident, if road users do not take measures; the traffic accident may be occurring. So we can use the intersection serious traffic collisions number to instead of the traditional traffic accident statistics method to evaluate traffic safety condition of the intersections.

\section{Grey clustering evaluation modals}

Grey clustering analysis firstly determine the Whitenization number of the different clusters index for clusters object, and then induct and classify base on a few grey, finally judge the clusters object to be-longing to which kind of grey. According to the principle of grey clustering, the mathematical model steps are as follows. 
The choice of evaluation index. TC represents the safety level of the intersection, which is traffic control, transportation facilities and the awareness of laws and regulations etc. Mixed Passenger Car Unit (MPCU) represents the traffic level of the intersection, which is traffic volume, traffic flow and the distribution of traffic signal etc. therefore, the paper adopts traffic conflict and the ratio of traffic conflicts and mixed passenger car unit (TC/MPCU) as evaluation index when we evaluate the traffic safety level of urban road intersection. This method has a high reliability.

The establishment of evaluation matrix. If the number of evaluation objects is $n$, the number of evaluation index items is $m$, evaluation object $i \in\{1,2, \ldots, n\}$, evaluation index $j \in\{1,2, \ldots, m\}$, assess sample matrix is dij, then

$$
D=d_{i j}=\left[\begin{array}{ccc}
d_{11} & \mathrm{~L} & d_{m} \\
\mathrm{M} & & \mathrm{M} \\
d_{n 1} & \mathrm{~L} & d_{n m}
\end{array}\right]
$$

To determine the grey tape and the whitenization value. The actual data of the evaluation are no dimensional; accordingly it was drawn that is the cumulative percentage frequency curve [6]. The whitenization values of the grey are the corresponding values of the different cumulative percent frequencies. For example, traffic safety condition is divided into four grey class level which are excellent (A), good (B), medium (C) and poor (D). The value of A, B, C, D will correspond to the cumulative per-cent frequencies of $15 \%, 40 \%, 60 \%, 80 \% . \lambda_{1}, \lambda_{2}, \lambda_{3}$ and $\lambda_{4}$ are whitenization value of four cumulative percent frequency points, see Figure 1.

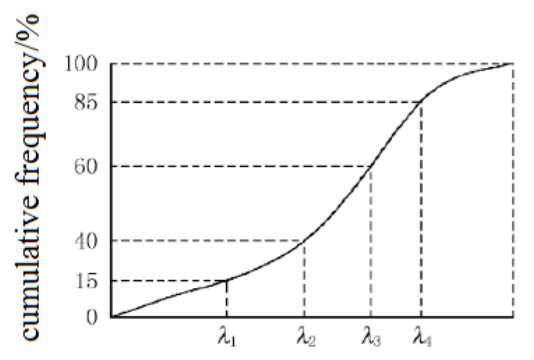

Whitenization value

Figure1. The cumulative percent frequency curve of evaluation index

To establish whitenization weight function of grey clustering. If $f_{j}(x)$ is whitenization weight function for grey clustering, the whitenization weight functions, which are corresponding to excellent, good, medium and poor of traffic safety condition [7,8], are shown in Figure 2.

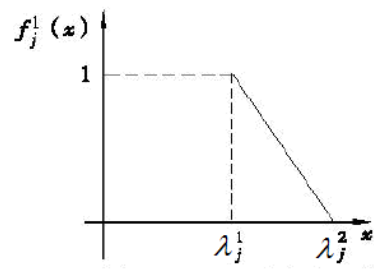

(a) The traffic safety condition is excellent
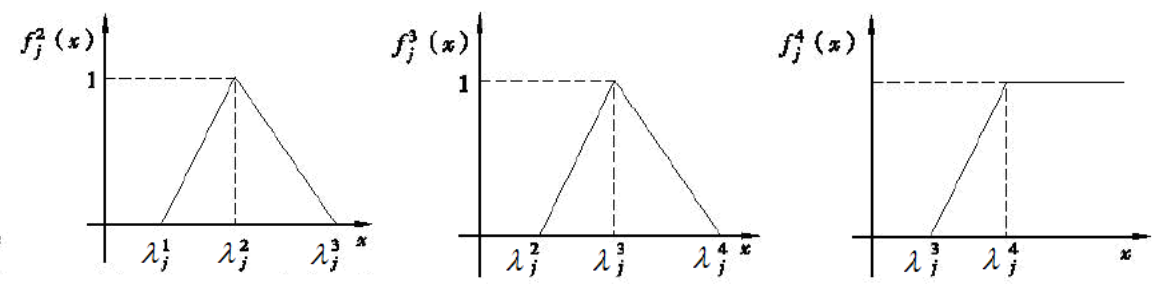

(b) The traffic safety condition is good

(c) The traffic safety

(d) The traffic safety condition is medium condition is poor

Figure 2. The general form of the whitenization function 
The grey clustering weight. If $\eta_{j t}$ is clustering weight that the $\mathrm{j}$ evaluation index belongs to the i grey tape, $\mathrm{t}$ is grey tape of the evaluation and $t \in\{1,2, \cdots, \mathrm{k}\}, \mathrm{k}$ is the number of grey tape, $\eta_{j t}$ can be obtained by formula (2).

$$
\eta_{j t}=\lambda_{j t} / \sum_{j=1}^{m} \lambda_{j t}
$$

Where, $\lambda_{j t}$ expresses whitenization value which the $j$ evaluation index belongs to the $t$ grey tape.

Grey clustering analysis. Clustering evaluation value $\sigma_{\mathrm{it}}$ of $\mathrm{t}$ grey class for the evaluation object $i$ can be calculated by formula (3).

$$
\sigma_{i t}=\sum f_{i t}\left(d_{i j}\right) \cdot \eta_{j t}
$$

If the grey clustering assessment sequence of the evaluation object $\mathrm{i}$ is $\sigma_{i k}^{*}=\left\{\sigma_{i 1}, \sigma_{i 2}, \mathrm{~L}, \sigma_{i k}\right\}$, the evaluation object belongs to $k^{*}$ gray class, which meet $\sigma_{i k}^{*}=\max \left(\sigma_{i 1}, \sigma_{i 2}, \mathrm{~L}, \sigma_{i k}\right)$, accordingly the safety condition level of clustering object can be determined.

\section{Case analysis}

To choose eight intersections of Baotou city as the research object, TC/MPCU value of each period was obtained through surveys, these observation periods include morning peak hour 7:30 8:30 (MPH), PM 15:30 16:30 and evening peak hour 17:30 18:30 (EPH) at the weekday. Survey results are shown in Table 1.

Table 1. TC and MPCU value of eight intersections in Baotou

\begin{tabular}{cccccccc}
\hline Intersetion & \multicolumn{2}{c}{ MPH } & \multicolumn{2}{c}{ Usual time } & \multicolumn{2}{c}{ EPH } \\
\cline { 2 - 7 } number & TC & MPCU & \multicolumn{2}{c}{ TC } & MPCU & TC & MPCU \\
\hline$\# 1$ & 5 & 4876 & 1 & 3452 & 5 & 5213 \\
$\# 2$ & 4 & 4501 & 3 & 3765 & 5 & 4100 \\
$\# 3$ & 10 & 5435 & 5 & 4569 & 11 & 5891 \\
$\# 4$ & 8 & 6783 & 7 & 4887 & 9 & 7123 \\
$\# 5$ & 15 & 7021 & 9 & 5431 & 13 & 7432 \\
$\# 6$ & 11 & 6789 & 5 & 5567 & 10 & 6430 \\
$\# 7$ & 12 & 6190 & 4 & 4890 & 14 & 7034 \\
$\# 8$ & 7 & 4012 & 3 & 3310 & 4 & 4512 \\
\hline
\end{tabular}

To determine the evaluation index. According to the previous description, the evaluation index value can be expressed with $D^{0}$, while $D^{*}$ is a dimensionless expression form of $D^{0}$. 


$$
D^{0}=\left|\begin{array}{lll}
0.0010 & 0.0003 & 0.0010 \\
0.0009 & 0.0008 & 0.0012 \\
0.0018 & 0.0011 & 0.0019 \\
0.0012 & 0.0014 & 0.0013 \\
0.0021 & 0.0017 & 0.0017 \\
0.0016 & 0.0009 & 0.0016 \\
0.0019 & 0.0006 & 0.0020 \\
0.0017 & 0.0012 & 0.0009
\end{array}\right| \quad D^{*}=\left|\begin{array}{lll}
0.4800 & 0.1748 & 0.4819 \\
0.4160 & 0.4808 & 0.6127 \\
0.8612 & 0.6604 & 0.9382 \\
0.5520 & 0.8644 & 0.6348 \\
1.0000 & 1.0000 & 0.8788 \\
0.7584 & 0.5420 & 0.7814 \\
0.9074 & 0.3702 & 1.0000 \\
0.8167 & 0.7292 & 0.4454
\end{array}\right|
$$

The characteristic value $\lambda_{i}$ of evaluation index.

$$
\lambda=\left|\begin{array}{llll}
0.4288 & 0.5933 & 0.8050 & 0.8797 \\
0.2139 & 0.4931 & 0.6367 & 0.7833 \\
0.4527 & 0.6171 & 0.7521 & 0.9026
\end{array}\right|
$$

To calculate weights of grey cluster. The weights of grey cluster can be calculated by formula 2 , the result is as follows.

$$
\eta_{j t}=\left|\begin{array}{llll}
0.3914 & 0.3483 & 0.3670 & 0.3429 \\
0.1953 & 0.2894 & 0.2902 & 0.3053 \\
0.4133 & 0.3623 & 0.3428 & 0.3518
\end{array}\right|
$$

Grey clustering weight matrix can be calculate by formula 3, the result is as follows.

$$
\sigma=\left|\begin{array}{llll}
0.4814 & 0.1727 & 0.0000 & 0.0000 \\
0.4111 & 0.6293 & 0.0000 & 0.0000 \\
0.0000 & 0.0000 & 0.3341 & 0.6592 \\
0.0982 & 0.5757 & 0.0449 & 0.3053 \\
0.0000 & 0.0000 & 0.0540 & 0.9445 \\
0.0000 & 0.2676 & 0.6611 & 0.0685 \\
0.0859 & 0.1621 & 0.0000 & 0.6947 \\
0.4133 & 0.0000 & 0.4167 & 0.2463
\end{array}\right|
$$

According to the equivalence principle of taking the larger value, $\sigma_{i k}^{*}=\max \left\{\sigma_{i 1}, \sigma_{i 2}, \mathrm{~L}, \sigma_{i k}\right\}$, the valuation object $\mathrm{i}$ belong to which category can be determined.

For example, $\sigma_{1 k}^{*}=\max \{0.4814,0.1727,0,0\}=0.4814=\sigma_{11}$, the safety level of intersection \#1 is excellent. Similarly, we can get the aforementioned eight intersections traffic safety level, which is \{excellent, good, poor, and good, poor, medium, and poor, medium \}. It can clearly be seen that traffic safety level of intersection 3, 5, 7 are poor and traffic conflicts are more, results are consistent with the actual traffic operation condition of the intersections. In order to reduce the traffic conflict numbers and to improve traffic safety and traffic capacity of the intersection, we should take some measures to strengthen usual management or take engineering measures to regulate running track of traffic flow. The method can be taken as follows: to adjust signal light settings make it more adaptable to local traffic conditions; to improve road traffic signs and markings of intersections; to set up traffic islands et al. 


\section{Conclusions}

(1) Example analysis demonstrate that traffic safety evaluation of intersection can overcome the problem of getting a lot of survey data and ensure a fair and objective evaluation results by traffic conflict techniques and grey clustering analysis.

(2) Method that determines whitenization function value has certain limitations by cumulative frequency curve method, therefore we need to explore new ways in future.

(3) The distance judgment between vehicles is not easy to grasp and survey accuracy is to be improved during traffic conflict survey.

(4) The security level of intersections can determine based on safety evaluation results of intersections. According to we can determine the order of intersection safety improvement and reasonably arrange construction funds.

\section{References}

[1] Huang Mingfang, Chen Tenglin, Evaluation of city traffic safety an intersection based on traffic conflict. Journal of Heilongjiang Institute of Technology. 27, 1 (2013) 10-13.

[2] Yang Yonghong, LV Dawei, Fu Xinsha, Traffic safety analysis and study of improvement measures for mountainous expressway. Hingway. 1 (2015) 94-99.

[3] Zhou Huabing, Xu Jianmin, Application of traffic conflict technology in assessing the intersection safety in Guilin. Western China. 1 (2008) 20-22.

[4] Ji Lina, Wang Fei and Cheng Wei, Study on evaluation of traffic safety based on TCT and gray clustering an intersection. Journal of Wuhan University of Technology (Transportation Science \& Engineering). 36, 3 (2012) 641-644.

[5] Cheng Wei, Theory and application of urban traffic conflict technology. Beijing: Science Press (2006)

[6] Zhu Xinglin, ELI Ismutulla and ARKEN Tohti, Grey cluster Method for highway safety evaluation. Highway Engineering. 34, 2 (2009) 157-160.

[7] Niu Huiyong, Study on safety evaluation of urban road traffic based on gray theory. China Safety Science Journal. 15, 9 (2005) 92-97.

[8] Dong Fenyi, Xiao Meidan, Liu Bin, Han Ying, Construction method of whitenization weight functions in grey system teaching. Journal of China Institute of Water Conservancy and Hydroelectric Power. 31, 3 (2010) 97-99. 